\title{
EMOTION TEACHING INTERFACE FOR FINGER BRAILLE EMOTION TEACHING SYSTEM
}

\author{
Yasuhiro Matsuda $^{1}$ and Tsuneshi Isomura ${ }^{1}$ \\ ${ }^{1}$ Department of Robotics and Mechatronics, Kanagawa Institute of Technology, \\ Atsugi, Japan \\ yasuhiroerm.kanagawa-it.ac.jp
}

\begin{abstract}
Finger Braille is one of the tactual communication methods utilized by deafblind individuals. Deafblind people who are skilled in Finger Braille can catch up with speech conversation and express various emotions by changing dotting strength and speed. In this paper, we designed the emotion teaching interface in order to express joy, sadness, anger and neutral for the Finger Braille emotion teaching system. We changed the previous background color (beige) of the teaching interface into 17 different colors. We also designed 8 kinds of dot patterns with different horizontal width and vertical length. The experiment to select the most suitable emotion teaching interfaces for joy, sadness, anger and neutral was conducted. The results showed that the dot patterns 6 (the wide and middle length pattern) or 1 (the small circle) with the lime, dark orange or yellow background colors are suitable for joy; the dot patterns 7 (the narrow and long pattern) or 4 (the narrow and middle length pattern) with the lavender, navy or blue background colors are suitable for sadness; the dot patterns 9 (the large circle) or 8 (the middle width and long pattern) with the red background color are suitable for anger; the dot pattern 5 (the middle circle) with the previous, honeydew, saddle brown or white background colors are suitable for neutral.
\end{abstract}

\section{KEYWORDS}

Emotional communication, Finger Braille, deafblind \& emotional color

\section{INTRODUCTION}

One of the most important factors of communication is emotion. Emotion is communicated in facial expressions, body movements, eye communication and paralanguage. Emotion is also affected by content of communication, particular objects and colors of environment. Colors are associated with particular emotions for human beings.

Deafblindness is a combination of varying degrees of both hearing and visual impairment. Deafblind people have difficulties with both verbal and emotional (nonverbal) communication. Deafblind people use many different communication media, depending on the age of onset of hearing and visual impairment and the available resources. Tactual communication is an important form of verbal and emotional communications for deafblind people.

David C. Wyld et al. (Eds) : CST, ITCS, JSE, SIP, ARIA, DMS - 2014

pp. 89-100, 2014. (C) CS \& IT-CSCP 2014

DOI : $10.5121 /$ csit.2014.4109 
"Yubi-Tenji" (Finger Braille) is one of the tactual communication media utilized by deafblind individuals (see Figure 1). In two-handed Finger Braille, the sender's index finger, middle finger and ring finger of both hands function like the keys of a Braille typewriter. The sender dots Braille code on the fingers of the receiver as if typing on a Braille typewriter. The receiver is assumed to recognize the Braille code. In one-handed Finger Braille, the sender first dots the left column of Braille code on the distal interphalangeal (DIP) joints of the three fingers of the receiver, and then the sender dots the right column of Braille code on the proximal interphalangeal (PIP) joints. Deafblind people who are skilled in Finger Braille communicate words and express various emotions because of the prosody (intonation) of Finger Braille [1]. The prosody of Finger Braille is a kind of paralanguage that helps the receiver recognize the dotted Braille code. The following were the features of the prosody: (1) the sender dots long at the end of the clause; (2) the sender pauses long after the end of clause; (3) the sender also dots long and strongly at the end of the sentence. However, non-disabled people generally are not skilled in Finger Braille. Consequently, deafblind people can communicate only through interpreters.
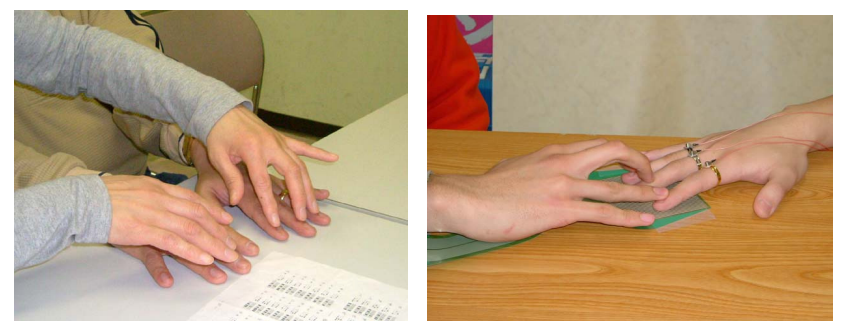

Figure 1. Two-handed Finger Braille (left) and one-handed Finger Braille (right)

Various Finger Braille input devices were developed. Amemiya et al. developed oboe-like Braille input interface (keyboard) [2]. An et al. developed Braille input gloves [3]. Fukumoto et al. developed a wearable input device with accelerometers mounted on the top of rings [4]. Hoshino et al. developed a Finger Braille input system that mounted accelerometers on the middle phalanges [5]. With these devices, deafblind people are burdened with wearing sensors, and they must master a new communication system.

We have recently been developing a Finger Braille support device which employs tactual communication. Figure 2 shows the concept of the Finger Braille support device. The advantages of this support device are as follows: (1) both deafblind people and non-disabled people unskilled in Finger Braille can communicate using conventional Finger Braille; (2) because the nondisabled people operate the support device and wear all of the sensors, deafblind people are not encumbered by the support device; (3) the intent of the support device is to assist both verbal and emotional communication.

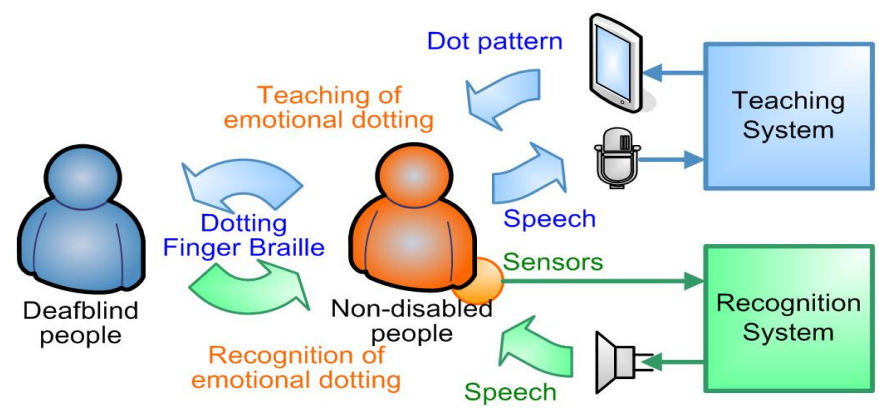

Figure 2. Concept of Finger Braille support device 
The support device consists of a Finger Braille (emotion) teaching system and a Finger Braille (emotion) recognition system. The teaching system recognizes the speech of a non-disabled person and displays the associated dot pattern of Finger Braille. The non-disabled person can then dot Finger Braille on the fingers of the deafblind person by observing the displayed dot pattern [6]-[7]. The emotion teaching system also teaches non-disabled person to express emotions. We have developed the teaching method of emotional expression using sentences about the impression of emotional expression [8].

The recognition system recognizes the dotting of Finger Braille by the deafblind person and synthesizes the associated speech for the non-disabled person [9]. We have developed the emotion recognition system which recognizes the emotions dotted by the deafblind person and presents the information for the non-disabled person [10].

The objective of this study is development of the emotion teaching interfaces to express joy, sadness, anger and neutral. In this paper we first designed the emotion teaching interfaces for the emotion teaching system. Then, an experiment to select the most suitable emotion teaching interfaces for joy, sadness, anger and neutral was conducted.

\section{DESIGN OF EMOTION TEACHING INTERFACE}

\subsection{Configuration of Teaching System}

The configuration of the teaching system was shown in Figure 3 [6]-[7]. First, a speech recognition (SR) engine recognizes the speech by the sender. Second, the teaching system converts the Kana script to Braille code by using the results of the speech recognition. Third, the teaching system retrieves the clause information by parsing the Braille code and segments the Braille code into clauses. Finally, the teaching system displays the associated dot pattern of the Braille code. The teaching system was developed on a tablet PC (Dell Latitude XT, CPU Core 2 Duo $1.33 \mathrm{GHz}$, RAM $2 \mathrm{~GB}, 12.1$ inch WXGA LCD with touch screen). The operating system was Microsoft Windows XP. The programming languages were Microsoft Visual Basic 6 and LPA WIN-PROLOG 4.500. The speech recognition engine was Microsoft Speech SDK (SAPI5.1). If the Braille code was not grammatically because of misrecognition of SR, the Braille code parser could not parse it. As a backup of the Braille code parser, we used Microsoft Global IME (Japanese) (IMM API).

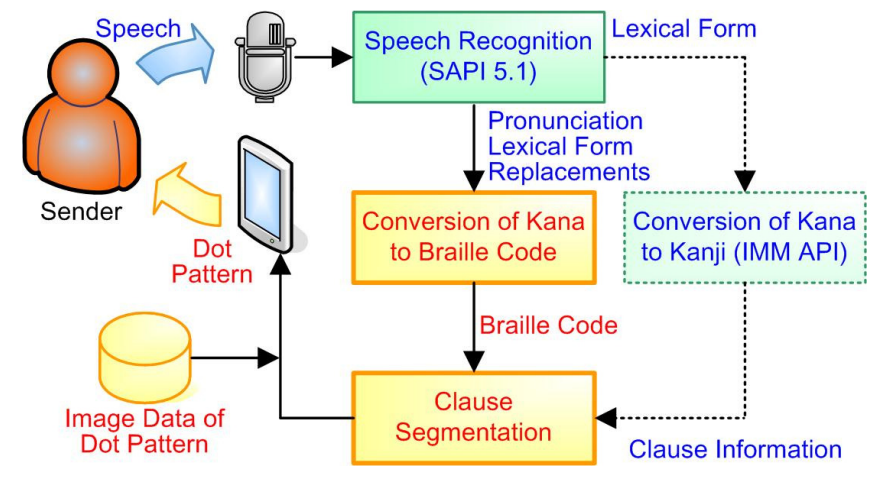

Figure 3. Configuration of the teaching system.

Figure 4 shows the previous teaching interfaces of the teaching system. The Braille code is displayed in the upper text box. The dot pattern is displayed in eighteen picture boxes (three columns and six rows). Clauses are displayed in the order of top to bottom of the first column, top 
to bottom of the second column, and top to bottom of the third column, according to the length of the clause. Sometimes a long clause is continued on the next page. If a clause has fewer than six characters, the next clause appears in the next column or the next page. In this way, the dot pattern of the clauses is displayed explicitly by the columns.

The red pattern in Figure 4 indicates the dotting of the DIP joints and the blue pattern indicates the dotting of the PIP joints. We designed three kinds of teaching interfaces for the sender. Teaching interface 1 displays the dot pattern illustrated on the fingers; teaching interface 2 displays only the dot pattern; teaching interface 3 displays the dot pattern with long and short arrows to indicate the duration of dotting. Teaching interface 1 is more symbolic and easier for beginners because they can see the dotting fingers. Teaching interface 2 has the most simplified signing and is suitable for the experienced senders. Teaching interface 3 teaches the duration of dotting to realize the non-disabled sender's prosodic dotting.

The buttons of speech recognition, edit, restatement, previous page and next page are located on the lower part of the display. The sender can touch the LCD directly to operate the teaching system and edit the Braille code.

In this study, we adopted one-handed Finger Braille using the right hand as the communication medium, because one-handed Finger Braille is easy to dot for non-disabled people and most human populations are right-handed (see Figure 5).
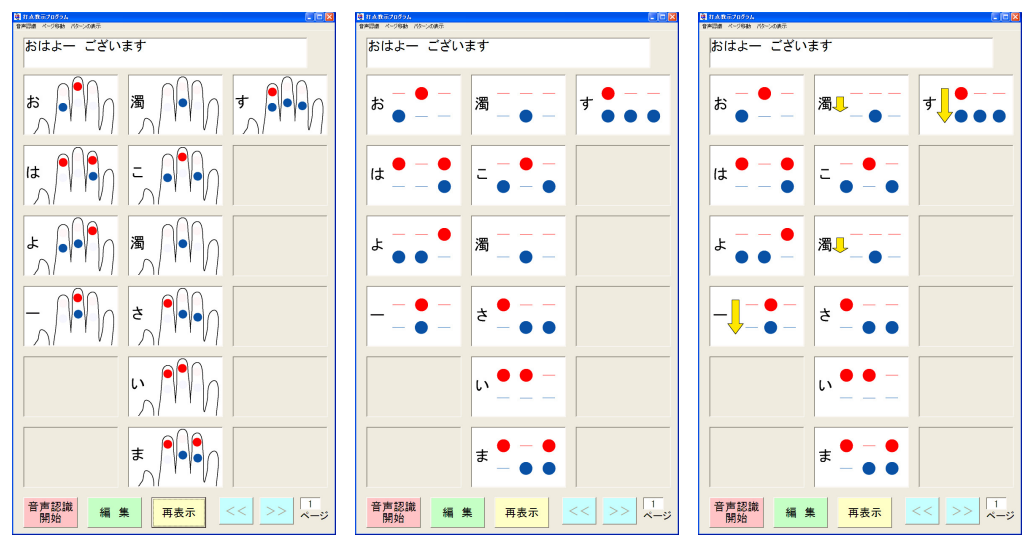

Figure 4. Three kinds of teaching interfaces: teaching interface 1 (left), teaching interface 2 (center) and teaching interface 3 (right). The displayed dot pattern is "Ohayo- / gozaimasu" ("Good morning").

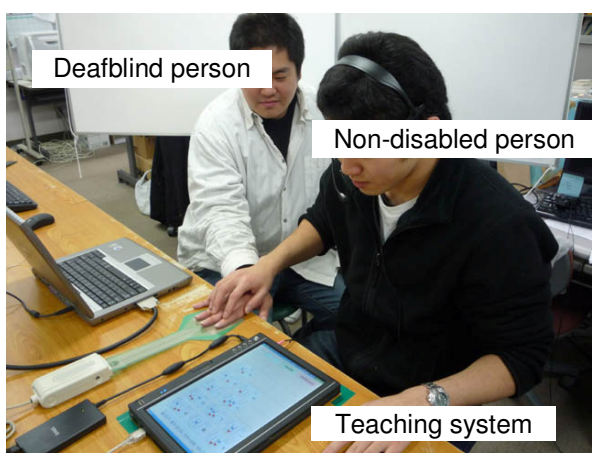

Figure 5. One-handed Finger Braille communication supported by the teaching system. 


\subsection{Teaching Method of Emotional Expression using Sentences}

In the previous study, we analyzed the features of emotional expression by Finger Braille interpreters [1]. The features were as follows: (1) the duration of the code of joy was significantly shorter than those of the other emotions (neutral, anger and sadness); (2) the duration of the code of sadness was significantly longer than those of the other emotions; (3) the finger load of anger was significantly larger than those of the other emotions; (4) the finger load of joy was significantly larger than those of sadness and neutral; (5) the duration of the code of anger was significantly shorter than those of sadness and neutral. We also analyzed the effectiveness of emotional expression and emotional communication between people unskilled in Finger Braille. The results indicate that the features and the impression of emotional expression by the unskilled people were very similar to those by the interpreters.

We noted the similarities of the impression of emotional expression between the interpreters and the unskilled people. We developed the teaching method using the sentences about the impression of emotional expression [8]. The teaching sentences of emotional expression that we developed were as follows.

Joy: Dot rhythmically.

Sadness: Dot weakly and slowly.

Anger: Dot strongly and little bit quickly.

Neutral: Dot politely and slowly with a constant rhythm.

The unskilled people read these sentences about the emotion which he/she want to express and then express the impression of emotional expression in the dotting of Finger Braille. The results of the evaluation experiment showed that the non-disabled subjects could express emotions better than the subjects who were not taught the features of emotional expression.

\subsection{Design of Emotion Teaching Interface}

Colors are associated with particular emotions for human beings, such as semantic words ("warm - cool", "heavy - light", "active - passive", etc.) or actual emotions [11]-[12]. In the present study, we designed the emotion teaching interface in order to express joy, sadness, anger and neutral, in addition to the teaching sentences of emotional expression. The concept of design is as follows: "the background color of the teaching interface will be associated with the emotion to express." We changed the previous background color (beige) of teaching interface 2 (see Figure 4) into 17 different colors. The designed teaching interfaces with 18 background colors are presented in Figure 9. The RGB triplets of the designed background colors are listed in Table 1. The color names are pursuant to the HTML color names.

Next, we modified the horizontal width and vertical length of the dot pattern of teaching interface 2. The concepts of modification are as follows: (1) "the wide dot pattern will be associated with the strong dotting and the narrow dot pattern will be associated with the weak dotting;" (2) "the long dot pattern will be associated with the long dotting duration and the short dot pattern will be associated with the short dotting duration." We designed 8 kinds of dot patterns with different horizontal width and vertical length (see Figure 10). Dot pattern 5 is the previous dot pattern (the middle circle). Dot pattern 1 is the small circle. Dot pattern 2 is the middle width and short pattern. Dot pattern 3 is the wide and short pattern. Dot pattern 4 is the narrow and middle length pattern. Dot pattern 6 is the wide and middle length pattern. Dot pattern 7 is the narrow and long pattern. Dot pattern 8 is the middle width and long pattern. Dot pattern 9 is the large circle. 
In the present study, we selected the most suitable combinations of the background color and dot pattern to express joy, sadness, anger and neutral through an experiment, as we discussed below.

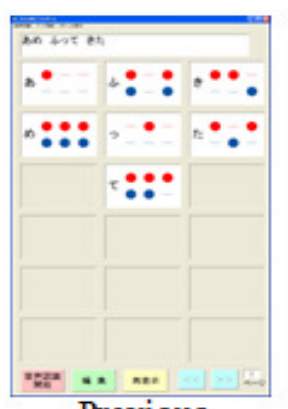

Previous

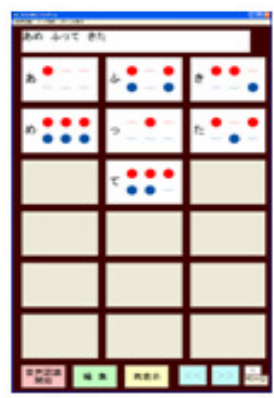

Maroon

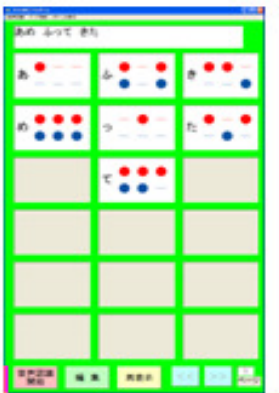

Lime

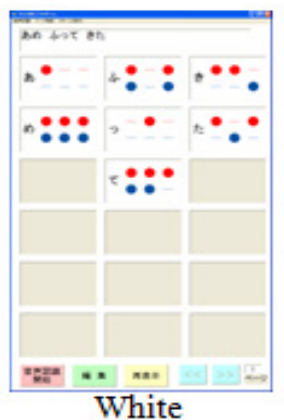

White

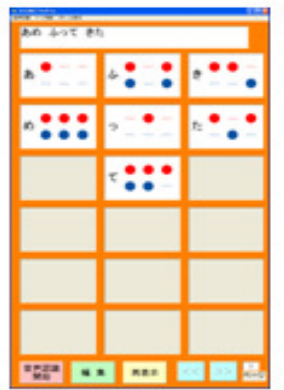

Dark Orange

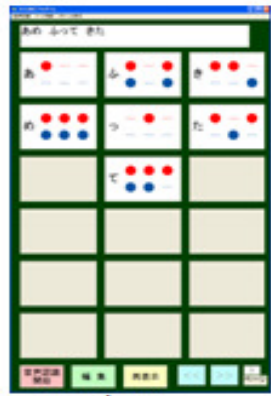

Dark Green

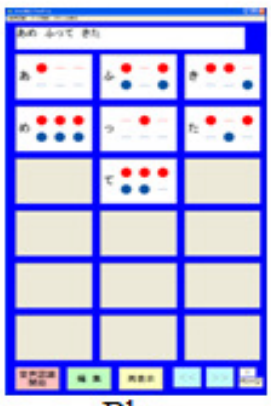

Blue
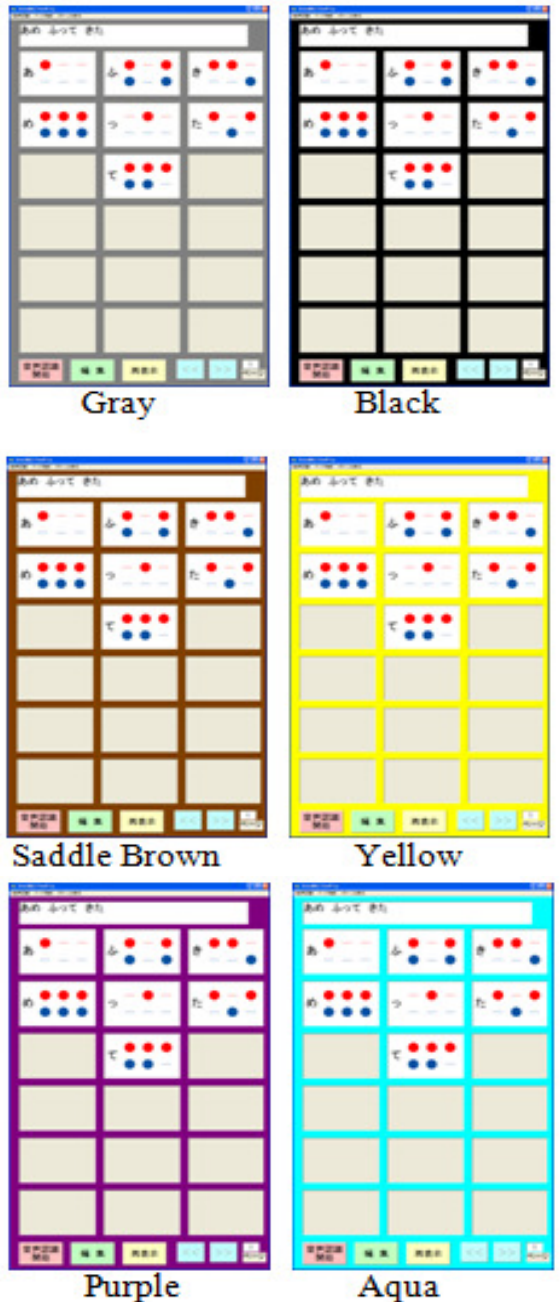

Yellow
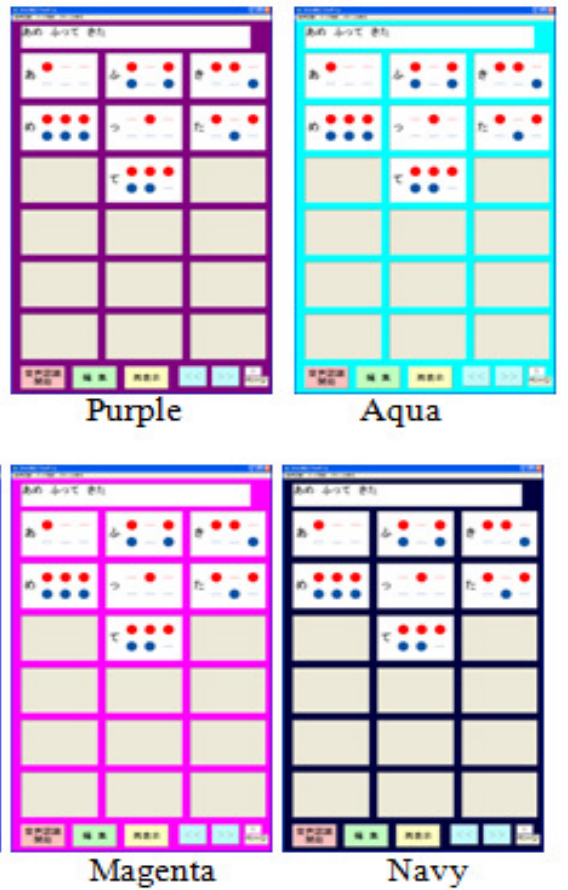
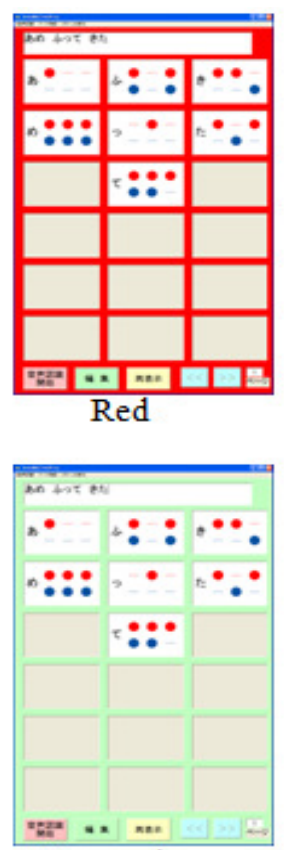

Honeydew

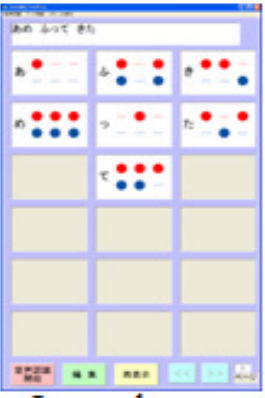

Lavender

Figure 6. Emotion Teaching interfaces with 18 background colors. The displayed dot pattern is "Ame / futte / kita" ("Rain has fallen"). 
Table 1. RGB triplets (hexadecimal) of designed background colors.

(Color names with * are darker than the associated HTML color names)

\begin{tabular}{|c|l|c|c|c|}
\hline No. & \multicolumn{1}{|c|}{ Color Name } & R & G & B \\
\hline 1 & Previous $\left(\right.$ Beige $^{*}$ ) & EC & E9 & D8 \\
\hline 2 & White & FF & FF & FF \\
\hline 3 & Gray & 80 & 80 & 80 \\
\hline 4 & Black & 00 & 00 & 00 \\
\hline 5 & Red & FF & 00 & 00 \\
\hline 6 & Maroon $*$ & 40 & 00 & 00 \\
\hline 7 & Dark Orange $*$ & FF & 80 & 00 \\
\hline 8 & Saddle Brown $*$ & 80 & 40 & 00 \\
\hline 9 & Yellow & FF & FF & 00 \\
\hline 10 & Honeydew $*$ & C0 & FF & C0 \\
\hline 11 & Lime & 00 & FF & 00 \\
\hline 12 & Dark Green $*$ & 00 & 40 & 00 \\
\hline 13 & Purple & 80 & 00 & 80 \\
\hline 14 & Aqua & 00 & FF & FF \\
\hline 15 & Lavender $*$ & C0 & C0 & FF \\
\hline 16 & Blue & 00 & 00 & FF \\
\hline 17 & Magenta & FF & 00 & FF \\
\hline 18 & Navy $*$ & 00 & 00 & 40 \\
\hline
\end{tabular}

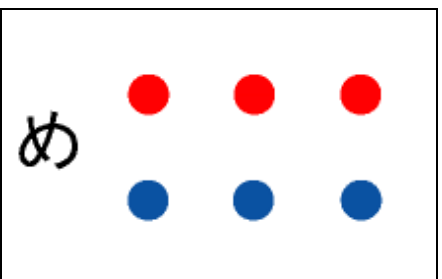

Dot pattern 1

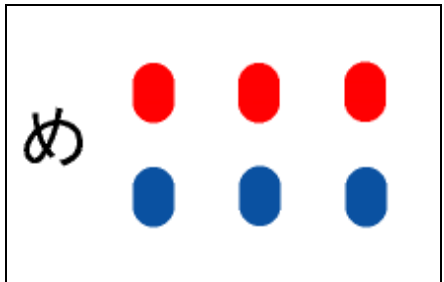

Dot pattern 4

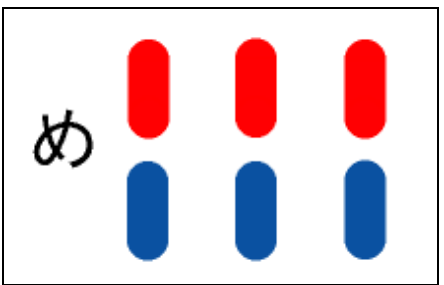

Dot pattern 7

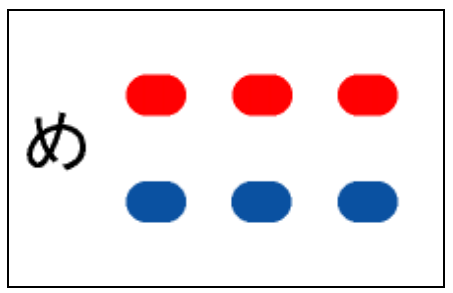

Dot pattern 2

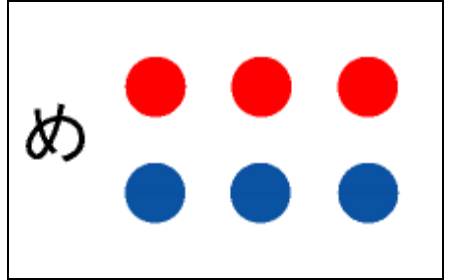

Dot pattern 5 (Previous)

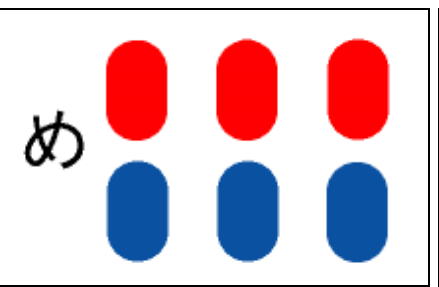

Dot pattern 8

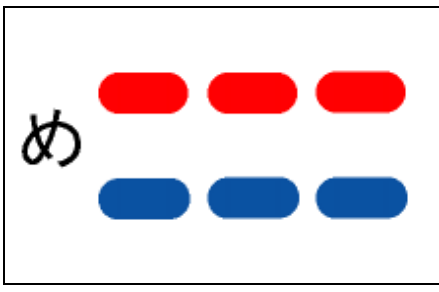

Dot pattern 3

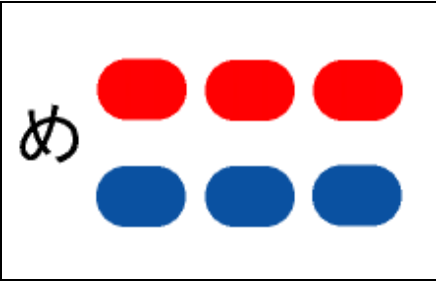

Dot pattern 6

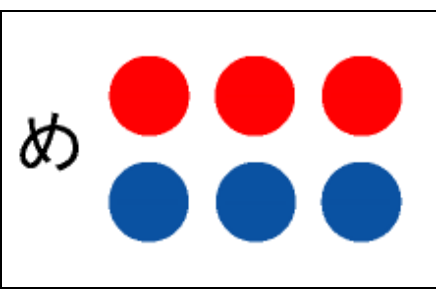

Dot pattern 9

Figure 7. Dot patterns with different width and length. 


\section{SELECTION EXPERIMENT}

\subsection{Methods}

To select the most suitable emotion teaching interfaces for joy, sadness, anger and neutral, a selection experiment was conducted.

The subjects were 14 male and 1 female college students (mean age $=22.6$, S.D. $=1.2$ ). The subjects have no experience of Finger Braille. At the beginning of the experiment, a tester described about Finger Braille, the teaching system and the objectives of this experiment. All subjects gave their informed consent after hearing a description of the study.

Two experimental sessions were conducted. In the session 1, the tester displayed one of the 18 emotion teaching interfaces of Figure 6. The dot pattern was the previous pattern (dot pattern 5 of Figure 7). By observing the displayed emotion teaching interface, the subject responded an associated emotion from "joy", "anger", "sadness", "fear", "disgust", "surprise" and "not applicable (NA)". These six emotions are the fundamental emotions of human being. The tester repeated displaying 18 emotion teaching interfaces with a predetermined random order.

In the session 2, the tester displayed one of the emotion teaching interfaces with the 9 dot patterns of Figure 7. The background color of the teaching interface was the previous color (beige). By observing the emotion teaching interface, the subject responded the impression about the dotting strength from "strongly", "weekly" and "not applicable (NA)"; and the impression about dotting duration from "long", "shortly" and "not applicable (NA)". The tester repeated displaying 9 emotion teaching interfaces with a predetermined random order.

The emotion teaching interfaces were displayed on an external 14 inches LCD (ThinkVision LT1421, Lenovo) placed in front of the subject. The tester operated a note PC (ProBook $4515 \mathrm{~s} / \mathrm{CT}, \mathrm{HP}$ ) to display the teaching interfaces (see Figure 8).

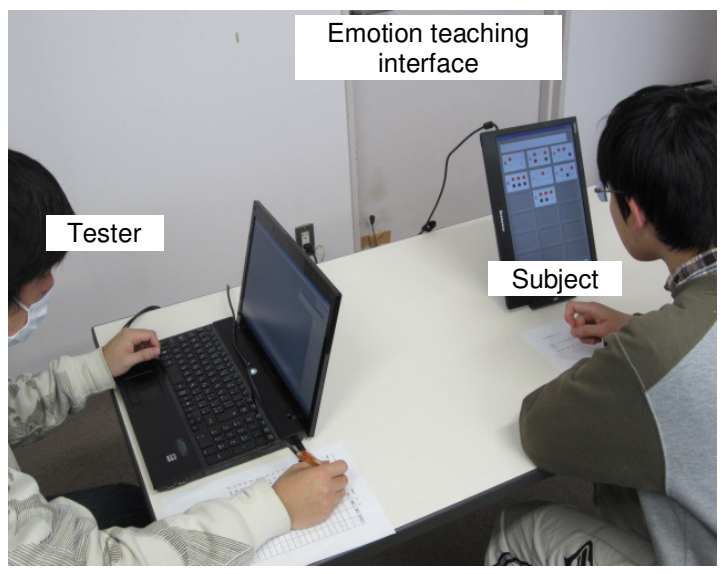

Figure 8. An experiment.

\subsection{Results}

Figure 9 shows the response ratio of emotions as a function of the background color of the emotion teaching interface. As a result, the associations of the lime, dark orange and yellow background colors with joy were common $(73 \%, 60 \%$ and $60 \%$, respectively). As for the red 
background color, $60 \%$ of subjects responded anger. The responses for the lavender, navy and blue background colors were very similar with sad $(60 \%, 53 \%$ and $47 \%$, respectively). About $47 \%$ of subjects regarded the black background color as fear. Almost all subjects (87\%) responded that the previous background color (beige) was not applicable (NA). A number of responses for the honeydew, saddle brown and white background colors were also NA (53\%, 47\% and $47 \%$, respectively).

Figure 10 shows the response ratio of dotting strength and duration as a function of the number of the dot pattern of the emotion teaching interface. As a result, a number of responses for dot patterns 1 and 2 were weakly (73\% and 53\%, respectively) and shortly (80\% and 53\%, respectively). Dot patterns 4 and 7 were mostly associated with weakly (53\% and 67\%, respectively) and long (60\% and $87 \%$, respectively). A number of responses for dot patterns 3,6 and 8 were strongly $(73 \%, 80 \%$ and $60 \%$, respectively) and long $(67 \%, 53 \%$ and $67 \%$, respectively). Dot pattern 9 was mostly associated with strongly (67\%) and shortly (47\%). A number of responses for dot pattern 5 were NA (47\% and 53\%).

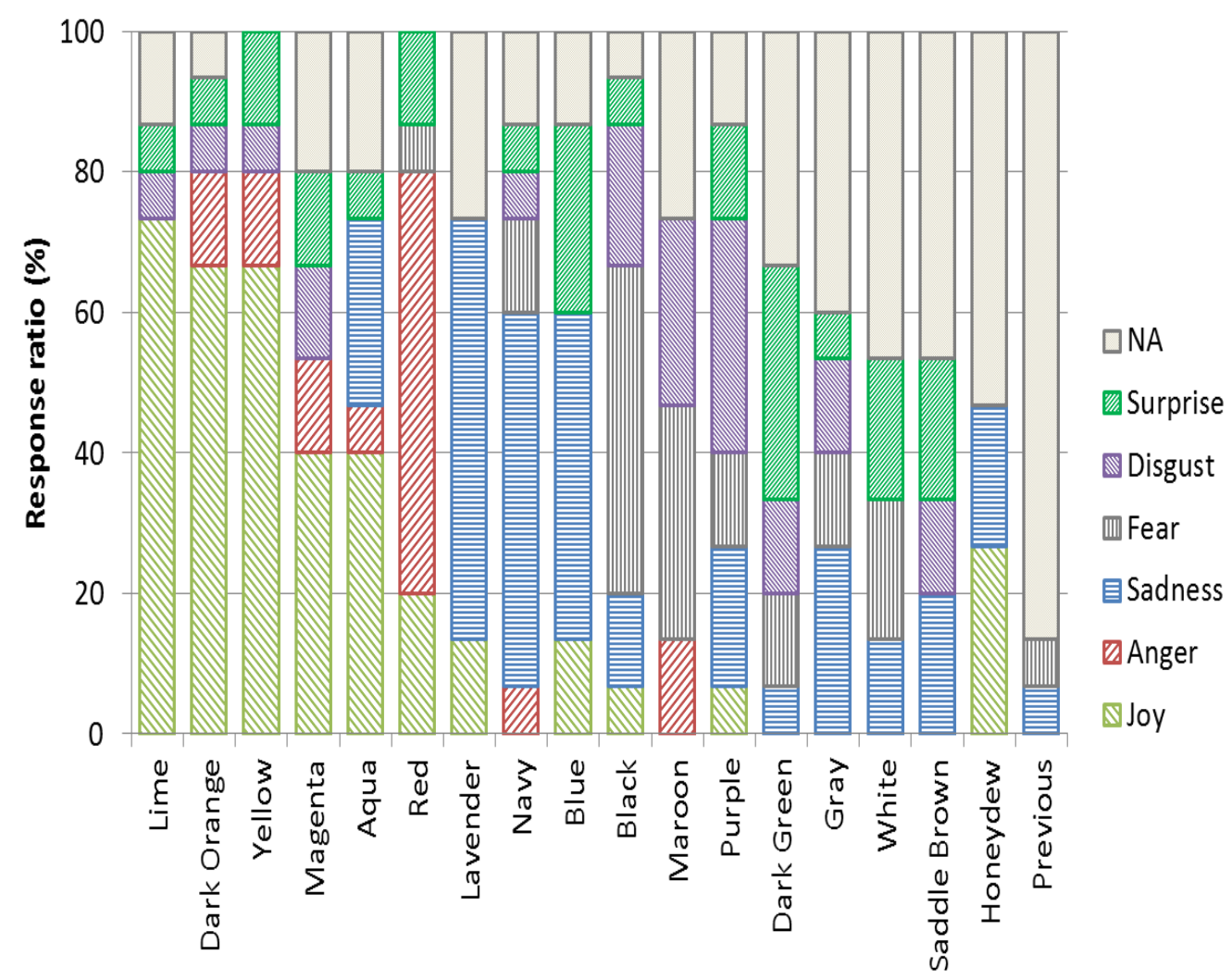

Background color of emotion teaching interface

Figure 9. Response ratio of emotions as a function of the background color of the emotion teaching interface. 


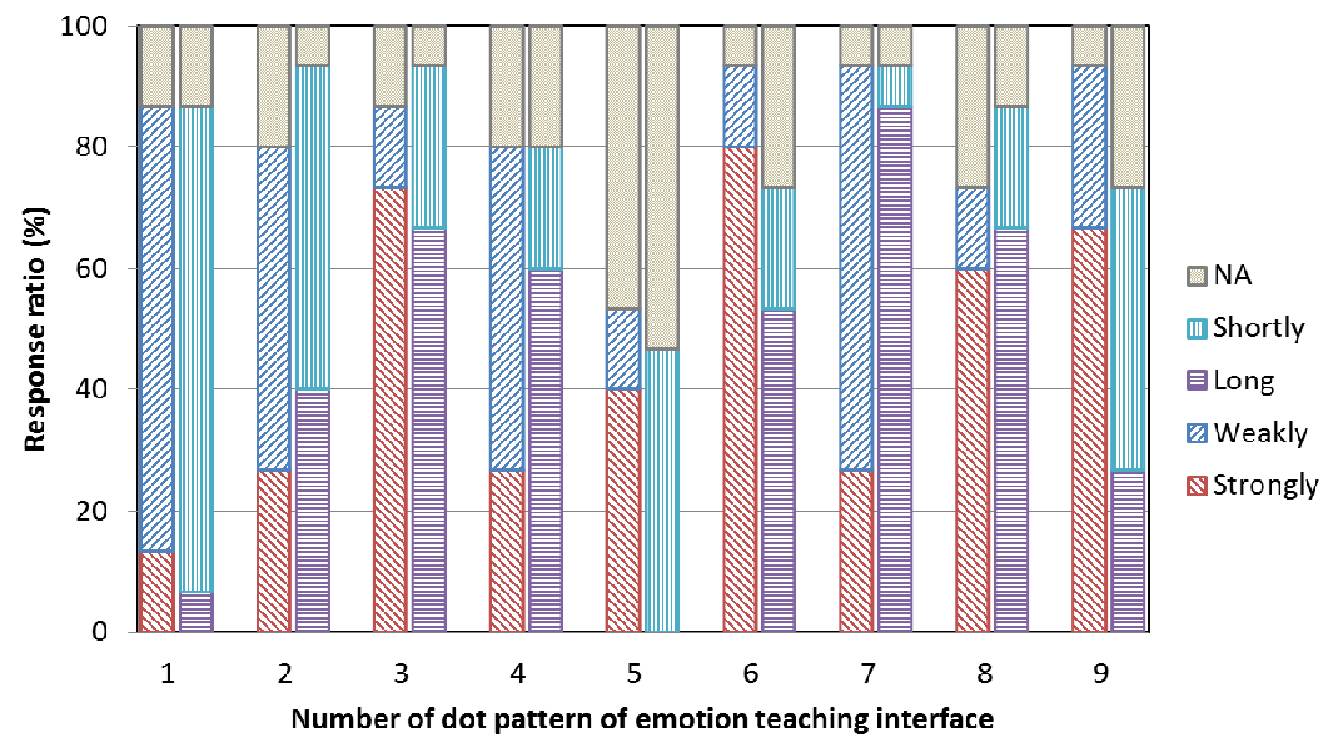

Figure 10. Response ratio of dotting strength and duration as a function of the dot pattern of the emotion teaching interface.

\subsection{Discussion}

According to the results of the session 1, the lime, dark orange and yellow background colors were associated with joy; the red background color was associated with anger; the lavender, navy and blue background colors were associated with sadness; the honeydew, saddle brown and white background colors were not associated with any emotions.

Clarke et al. investigated the relationship between colors and emotions [11]. They revealed that orange and yellow are associated with joy; red is associated with anger; blue is associated with sadness; gray is not associated with any emotions. These results were similar to our experimental results.

According to the results of the session 2, dot patterns 1 and 2 were associated with weak and short dotting. Dot patterns 4 and 7 were associated with weak and long dotting. Dot patterns 3, 6 and 8 were associated with strong and long dotting. Dot pattern 9 was associated with strong and short dotting. As for the concept of design of the dot patterns, dot pattern 3 and 6 should be associated with strong and short dotting. The other results were similar to the concept of the design.

As mentioned above, joy was characterized by little bit strong and short dotting. Sadness was characterized by weak and long dotting. Anger was characterized by strong and little bit short dotting. Neutral was characterized constant dotting without emotion. Thus, we conclude that the most suitable teaching interfaces for joy, sadness, anger and neutral are as follows.

Joy: Dot patterns 6 or 1 with the lime, dark orange or yellow background colors. Sadness: Dot patterns 7 or 4 with the lavender, navy or blue background colors. Anger: Dot patterns 9 or 8 with the red background color.

Neutral: Dot pattern 5 with the previous, honeydew, saddle brown or white background colors. 


\section{CONCLUSIONS}

In this paper, we designed the emotion teaching interface in order to express joy, sadness, anger and neutral for the Finger Braille emotion teaching system. We changed the previous background color (beige) of the teaching interface into 17 different colors. We also designed 8 kinds of dot patterns with different horizontal width and vertical length. The experiment to select the most suitable emotion teaching interfaces for joy, sadness, anger neutral and was conducted. The results showed that dot patterns 6 (the wide and middle length pattern) or 1 (the small circle) with the lime, dark orange or yellow background colors are suitable for joy; dot patterns 7 (the narrow and long pattern) or 4 (the narrow and middle length pattern) with the lavender, navy or blue background colors are suitable for sadness; dot patterns 9 (the large circle) or 8 (the middle width and long pattern) with the red background color is suitable for anger; dot pattern 5 (the middle circle) with the previous, honeydew, saddle brown or white background colors are suitable for neutral.

Our future plans are: (1) verification of the impression of the combinations of the background color and dot pattern; (2) evaluation of the emotional expression using these emotion teaching interfaces.

\section{ACKNOWLEDGEMENTS}

This work was supported by JSPS KAKENHI Grant Number 25350683.

\section{REFERENCES}

[1] Y. Matsuda, I. Sakuma, Y. Jimbo, E. Kobayashi, T. Arafune \& T. Isomura, (2010) "Emotional Communication in Finger Braille", Advances in Human-Computer Interaction, Vol. 2010, Article ID 830759, 23 pages.

[2] S.S. An, J.W. Jeon, S. Lee, H. Choi \& H.G. Choi, (2004) "A Pair of Wireless Braille-Based Chording Gloves", Proceedings of 9th International Conference on Computers Helping People with Special Needs, pp.490-497.

[3] T. Amemiaya, K. Hirota \& M. Hirose, (2004) "OBOE: Oboe-Like Braille Interface for Outdoor Environment", Proceedings of 9th International Conference on Computers Helping People with Special Needs, pp.498-505.

[4] M. Fukumoto \& Y. Tonomura, (1997) "Body Coupled FingeRing: Wireless Wearable Keyboard", Proceedings of the ACM Conference on Human Factors in Computing Systems, pp.147-154.

[5] T. Hoshino, T. Otake \& Y. Yonezawa,(2002) "A Study on a Finger-Braille Input System Based on Acceleration of Finger Movements", IEICE Transactions on Fundamentals of Electronics, Communications and Computer Sciences, Vol.J85-A, no.3, pp.380-388.

[6] Y. Matsuda \& T. Isomura, (2010) "Finger Braille Teaching System", Character Recognition, Sciyo, pp.173-188.

[7] Y. Matsuda \& T. Isomura, (2011) "Improvement of Interfaces of Finger Braille Teaching System", Journal of Computer and Information Technology, Vol. 1, No. 2, pp.19-28.

[8] Y. Matsuda \& T. Isomura, (2013) "Development of Teaching Method of Emotional Expression using Finger Braille", Journal of Communication and Computer, Vol. 10, No. 4, pp.567-577.

[9] Y. Matsuda \& T. Isomura, (2012) "Finger Braille Recognition System", Advances in Character Recognition, InTech, pp.193-210.

[10] Y. Matsuda \& T. Isomura, (2012) "Emotion Recognition System of Finger Braille", International Review on Computers and Software (I.RE.CO.S), Vol. 7, No. 7, pp.3494-3501.

[11] T. Clarke \& A. Costall, (2008) "The Emotional Connotations of Color: A Qualitative Investigation", Color Research and Application, Vol. 33, No. 5, pp.406-410.

[12] M. Solli \& R. Lenz, (2011) "Color Emotions for Multi-Colored Images", Color Research and Application, Vol. 36, No. 3, pp.210-217. 


\section{AUTHOR}

\section{Yasuhiro Matsuda}

Professor in the Department of Robotics and Mechatronics, Kanagawa Institute of Technology. He obtained Ph.D. degree in environmental studies from the University of Tokyo in 2007. His research interests include assistive technology, human interface and emotional communication. He is a member of IEEE Engineering in Medicine and Biology Society.

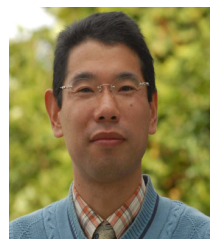

